\title{
Illustrating MIMO transmission by means of sound waves
}

\author{
Luis Mendo \\ Signals, Systems and Radiocommunications Dept. \\ Technical University of Madrid \\ E-mail: luis.mendo@upm.es
}

April 7, 2016

\begin{abstract}
This paper shows how the fundamental concepts of MIMO transmission can be illustrated using cheap audio equipment. Experiments are proposed which cover both diversity and spatial multiplexing techniques. Regarding the former, MRC receive diversity and Alamouti transmit diversity are examined. For the latter, V-BLAST and SVD-based methods are demonstrated, and their functioning is compared. The experiments allow the students to observe the most important features of MIMO, and can be helpful for wireless communication courses.

Keywords: Communication engineering education; MIMO; spatial multiplexing; diversity; multipath channels; acoustic propagation; electromagnetic propagation; electrical engineering education
\end{abstract}

\section{Introduction}

Multiple-Input Multiple-Output (MIMO) techniques involve the use of multiple antennas at the receiver, at the transmitter, or both, in order to improve certain characteristics of the transmission in multipath channels. All recent mobile communication standards, such as LTE (3GPP Long-Term Evolution), IEEE 802.11 (Wi-Fi) and IEEE 802.16 (WiMAX), rely on MIMO as a capacity-enhancing technique [2].

MIMO techniques can be classified into two groups, depending on their purpose: power/diversity gain or spatial multiplexing. In the following, each of the antennas that form an array will be referred to as antenna element; and $n_{\mathrm{t}}$ and $n_{\mathrm{r}}$ will denote the number of antenna elements at the transmitting and receiving arrays respectively.

At the basis of MIMO are abstract mathematical concepts, some of which may be difficult to grasp by students. It is thus desirable for the teacher to be able to illustrate those concepts in a more concrete way. The aim of the paper is to present some methods that achieve this, both for power/diversity gain and for spatial multiplexing.

In a similar mood as [3], sound waves are used for this purpose. Their main advantage over radio waves is that sound can be transmitted and received using cheap 
equipment: loudspeakers, microphones and laptop computers. A second benefit is that sound can be directly perceived, which brings increased realism. Use of sound instead of radio is possible by virtue of the existing analogies between both phenomena; in particular, the two types of waves experience multipath propagation.

Standard laptop sound cards have stereo outputs and inputs, and thus MIMO systems up to $2 \times 2$ can be set up. Higher-order systems do not involve essential new concepts, and therefore the restriction $n_{\mathrm{t}}, n_{\mathrm{r}} \leq 2$ is not significant.

A summary of MIMO concepts and notation is given in Section 2. The required equipment is specified in Section 3. The experiments are described in Sections 4 and 5 , which deal respectively with power gain measurements and spatial multiplexing measurements. Conclusions are presented in Section 6.

\section{Basic MIMO concepts and notation}

All MIMO techniques to be described consist of a spatial pre-processing (or "precoding") at the transmitter and a spatial post-processing at the receiver.

The propagation channel is described by an $n_{\mathrm{r}} \times n_{\mathrm{t}}$ channel matrix $\mathbf{H}$, whose $(i, j)$ entry $h_{i j}$ is the complex gain from the symbol fed to the $j$-th transmitting array element to that observed at the $i$-th receiving array element. The number $h_{i, j}$ includes, in addition to propagation effects, the transformation between symbols and waveforms.

\subsection{Power gain and diversity gain}

In this type of MIMO transmission, the pre-processing or post-processing steps aim at maximizing the array gain in the direction to / from the other end. This results in increased SNR (power gain) and less pronounced fading (diversity gain).

At the receiver side, with $n_{\mathrm{t}}=1, n_{\mathrm{r}}>1$, use of this technique requires determining the complex array weights to be applied. Let $x$ denote the transmitted symbol, and let $\mathbf{y}$ be the $n_{\mathrm{r}} \times 1$ vector of received symbols, such that the symbol at the $i$-th receiving array element is $y_{i}$. Then $y_{i}=h_{i, 1} x$ (note that $\mathbf{H}$ has size $n_{\mathrm{r}} \times 1$ ). Assuming equal noise power at all antenna elements, the optimum weights are $h_{i, 1}$, corresponding to maximal-ratio combining (MRC) [4, chapter 3]; and the combined received symbol, $y$, is ${ }^{1}$

$$
y=h_{1,1}^{*} y_{1}+\cdots+h_{n_{\mathrm{r}}, 1}^{*} y_{n_{\mathrm{r}}}=\left(\left|h_{1,1}\right|^{2}+\cdots+\left|h_{n_{\mathrm{r}}, 1}\right|^{2}\right) x .
$$

As can be seen, to compute $y$ the receiver needs to estimate the channel gains $h_{i, 1}$.

In order to use this approach at the transmitter, assuming $n_{\mathrm{t}}>1, n_{\mathrm{r}}=1$, the receiver would have to estimate and feed back the $n_{\mathrm{t}}$ complex values $h_{1, j}$. For $n_{\mathrm{t}}=2$, an alternative technique proposed by Alamouti [1] achieves the same performance while avoiding feedback. For each pair of consecutive symbol periods $m$ and $m+1$, let $x[m], x[m+1]$ denote the source symbols at the transmitter. According to the Alamouti method, the transmitting antenna element 1 is fed with the transformed symbols $\bar{x}_{1}[m]=x[m]$ and $\bar{x}_{1}[m+1]=-x^{*}[m+1]$, whereas the antenna element 2 is fed with $\bar{x}_{2}[m]=x[m+1]$ and $\bar{x}_{2}[m+1]=x^{*}[m]$. The complex channel gains from the transmitting antenna elements to the receiving antenna, $h_{1,1}$ and $h_{1,2}$, are assumed to be

\footnotetext{
${ }^{1}$ The notation $z^{*}$ represents the complex conjugate of a number $z$.
} 


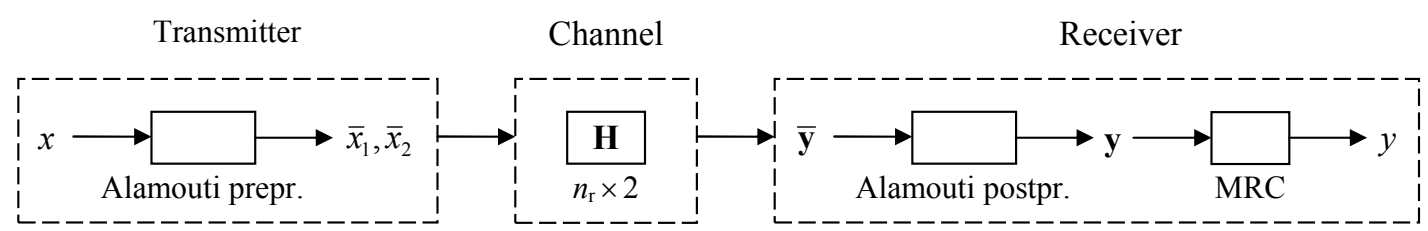

Figure 1: Signal processing for Alamouti transmit diversity and receive MRC

the same for periods $m$ and $m+1$. The received symbols are, by superposition,

$$
\bar{y}[m]=h_{1,1} x[m]+h_{1,2} x[m+1], \quad \bar{y}[m+1]=-h_{1,1} x^{*}[m+1]+h_{1,2} x^{*}[m] .
$$

The receiver post-processing consists in computing

$$
y[m]=h_{1,1}^{*} \bar{y}[m]+h_{1,2} \bar{y}^{*}[m+1], \quad y[m+1]=h_{1,2}^{*} \bar{y}[m]-h_{1,1} \bar{y}^{*}[m+1],
$$

which again requires the receiver to estimate $h_{1,1}$ and $h_{1,2}$. From (2) and (3),

$$
y[m]=\left(\left|h_{1,1}\right|^{2}+\left|h_{1,2}\right|^{2}\right) x[m], \quad y[m+1]=\left(\left|h_{1,1}\right|^{2}+\left|h_{1,2}\right|^{2}\right) x[m+1],
$$

which is the same as given in (1) for MRC (with the obvious interchange of subindices).

The Alamouti technique can be combined with MRC. Let $\overline{\mathbf{y}}[m], \overline{\mathbf{y}}[m+1]$ denote the $n_{\mathrm{r}} \times 1$ vectors of received symbols at periods $m$ and $m+1$. The receiver computes $n_{\mathrm{r}} \times 1$ vectors $\mathbf{y}[m], \mathbf{y}[m+1]$, where $y_{i}[m]$ and $y_{i}[m+1]$ are obtained as in (3):

$$
y_{i}[m]=h_{i, 1}^{*} \bar{y}_{i}[m]+h_{i, 2} \bar{y}_{i}^{*}[m+1], \quad y_{i}[m+1]=h_{i, 2}^{*} \bar{y}_{i}[m]-h_{i, 1} \bar{y}_{i}^{*}[m+1],
$$

which gives

$$
y_{i}[m]=\left(\left|h_{i, 1}\right|^{2}+\left|h_{i, 2}\right|^{2}\right) x[m], \quad y_{i}[m+1]=\left(\left|h_{i, 1}\right|^{2}+\left|h_{i, 2}\right|^{2}\right) x[m+1] .
$$

Lastly, MRC is applied to $y_{1}[m], \ldots, y_{n_{\mathrm{r}}}[m]$ to obtain a single $y[m]$ as in (1), and similarly for symbol period $m+1$. Figure 1 summarizes the process.

When both Alamouti transmit diversity and receive MRC are used, the terms $\left|h_{i, 1}\right|^{2}+\left|h_{i, 2}\right|^{2}, i=1, \ldots, n_{\mathrm{r}}$ in (6) can be interpreted as coefficients of an "equivalent channel" formed by the actual propagation channel and the Alamouti processing; and they are real because the Alamouti post-processing compensates for the channel phase.

\subsection{Spatial multiplexing}

If multiple antennas are available at transmitter and receiver, several streams can be spatially multiplexed using different beams. Two conditions need to be fulfilled:

1. The transmitted beams should point in sufficiently separated directions, so that they do not overlap significantly.

2. The multipath environment should provide a large number of obstacles ("rich scattering"), so that the transmitted streams can arrive at the receiver from directions sufficiently separated to avoid overlapping between receiver beams. 


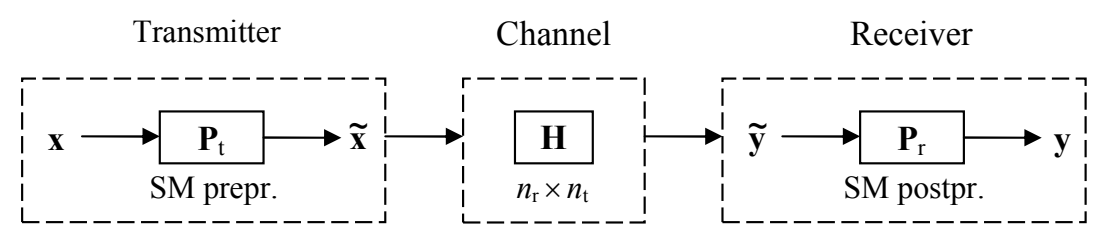

Figure 2: Signal processing for spatial multiplexing

The number of spatial streams will be denoted as $n$. In the rest of this section the dependency on the time index will be dropped for convenience.

At the transmitter, each spatial stream is mapped to the $n_{\mathrm{t}}$ antenna elements using linear spatial pre-processing. Specifically, the original symbols, denoted by an $n \times 1$ vector $\mathbf{x}$, are transformed into an $n_{\mathrm{t}} \times 1$ vector $\tilde{\mathbf{x}}=\mathbf{P}_{\mathrm{t}} \mathbf{x}$, where $\mathbf{P}_{\mathrm{t}}$ is the $n_{\mathrm{t}} \times n$ complex pre-processing matrix. The vector $\tilde{\mathbf{x}}$ is fed to the transmitting array.

Similarly, let the $n_{\mathrm{r}} \times 1$ vector $\tilde{\mathbf{y}}=\mathbf{H} \tilde{\mathbf{x}}$ denote the symbols observed at the receiving array. An $n \times n_{\mathrm{r}}$ post-processing matrix $\mathbf{P}_{\mathrm{r}}$ is applied ${ }^{2}$ to yield the $n \times 1$ vector

$$
\mathbf{y}=\mathbf{P}_{\mathrm{r}} \tilde{\mathbf{y}}=\mathbf{P}_{\mathrm{r}} \mathbf{H} \mathbf{P}_{\mathrm{t}} \mathbf{x} .
$$

With a suitable choice of matrices $\mathbf{P}_{\mathrm{t}}$ and $\mathbf{P}_{\mathrm{r}}$, the vector $\mathbf{y}$ will be a good estimation of $\mathbf{x}$. Figure 2 illustrates these operations.

Finding the optimum $\mathbf{P}_{\mathrm{t}}$ and $\mathbf{P}_{\mathrm{r}}$ matrices requires that the receiver estimates the channel matrix $\mathbf{H}$ and computes its singular-value decomposition (SVD) [4, section 7.1]. This is given as ${ }^{3} \mathbf{H}=\mathbf{U} \Lambda \mathbf{V}^{*}$, where $\mathbf{U}$ and $\mathbf{V}$ are unitary ${ }^{4}$ matrices of sizes $n_{\mathrm{r}} \times n_{\mathrm{r}}$ and $n_{\mathrm{t}} \times n_{\mathrm{t}}$ respectively, and $\Lambda$ is an $n_{\mathrm{r}} \times n_{\mathrm{t}}$ matrix whose off-diagonal elements are zero. Let $n_{\mathrm{s}}=\min \left\{n_{\mathrm{t}}, n_{\mathrm{r}}\right\}$. The diagonal elements of $\Lambda$, denoted as $\lambda_{1}, \ldots, \lambda_{n_{\mathrm{s}}}$, are the singular values of $\mathbf{H}$, which satisfy $\lambda_{1} \geq \lambda_{2} \geq \ldots \geq \lambda_{n_{\mathrm{s}}} \geq 0$. The number of non-zero singular values is the rank of $\mathbf{H}$, and will be denoted as $r$.

Using $\mathbf{P}_{\mathrm{t}}=\mathbf{V}, \mathbf{P}_{\mathrm{r}}=\mathbf{U}^{*}$, and assuming all singular values are non-zero, $n_{\mathrm{s}}$ noninterfering streams are obtained, and the total gain for the $k$-th stream is $\lambda_{k}$. Indeed from (7), since $\mathbf{U}$ and $\mathbf{V}$ are unitary, $\mathbf{y}=\mathbf{U}^{*} \mathbf{H V x}=\Lambda \mathbf{x}$. This requires feedback of channel information, namely of $\mathbf{V}$, to the transmitter. A measure of the disparity of the gains experienced by the spatial streams is given by the condition number, $\lambda_{1} / \lambda_{n_{\mathrm{s}}}$.

If some of the singular values are zero or very small, only the spatial streams associated with the largest values should be transmitted. This involves additional feedback. In general, the restriction $n \leq r \leq n_{\mathrm{s}}$ applies for any choice of $\mathbf{P}_{\mathrm{t}}$ and $\mathbf{P}_{\mathrm{r}}$.

Spatial multiplexing can also be achieved without feedback of channel information. Each stream is transmitted using fixed beams; and is received with interference from other streams, which should be canceled based on an estimation of $\mathbf{H}$. A possible approach, assuming $n=n_{\mathrm{t}}=n_{\mathrm{r}}$ and $\mathbf{H}$ non-singular, is to set $\mathbf{P}_{\mathrm{t}}=\mathbf{I}$ at the transmitter. This is the V-BLAST (Vertical-Bell Laboratories Layered Space-Time) scheme [4, section 3.3]. The receiver can then apply $\mathbf{P}_{\mathrm{r}}=\mathbf{H}^{-1}$ to obtain, according to (7), $\mathbf{y}=\mathbf{H}^{-1} \mathbf{H I x}=\mathbf{x}$. In practice $\mathbf{H}$, considered as a random matrix, will be non-singular almost surely; however, it may be ill-conditioned, which may degrade performance.

\footnotetext{
${ }^{2}$ Receiver post-processing need not be linear in general. However, focus will be restricted to linear post-processing, because it is widely used in practice and is sufficient for illustrating the main ideas.

${ }^{3}$ For a matrix $\mathbf{M}$, the notation $\mathbf{M}^{*}$ indicates its conjugate transpose.

${ }^{4} \mathrm{~A}$ square matrix $\mathbf{M}$ is unitary if $\mathbf{M} \mathbf{M}^{*}=\mathbf{M}^{*} \mathbf{M}=\mathbf{I}$.
} 


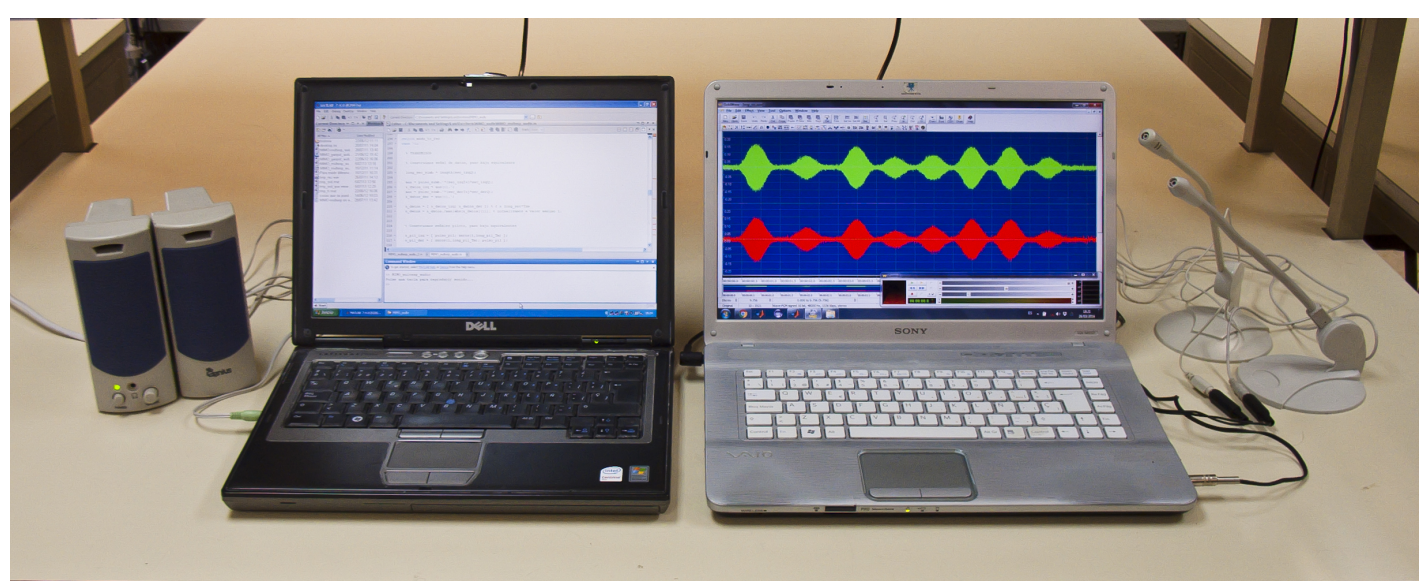

Figure 3: Equipment used in the measurements

\section{Necessary equipment}

The equipment is similar to that in [3]. Two laptop computers are used as transmitter and receiver respectively. A pair of loudspeakers is required at the transmitter. The computer used as receiver needs to have a stereo recording input, to which two mono microphones are connected. This may require an adapter plug or cable. The loudspeakers and microphones need not be of particularly good quality.

Transmitter processing and sound generation are done in Matlab. At the receiver a standard audio editor is used for recording. The stereo signal picked by the two microphones is saved to an audio file, which is then read and processed using Matlab.

Figure 3 shows the equipment used for the example measurements described in this paper. The transmitting computer appears on the left, with the loudspeakers connected to its sound output on the left-hand side, and running the Matlab program for transmitter processing and sound reproduction. On the right is the receiving computer, with a pair of microphones connected by means of an adapter cable to the recording input on the right-hand side. The computer is running Matlab (not shown) and GoldWave, an audio editor for recording the stereo received signal.

\section{Power gain measurements}

The measurements in this and the following sections will be carried out in timeinvariant channels. This simplifies channel estimation, as it suffices to do it once at the beginning of the measurement. All objects or people should remain static for the duration of the measurement (which is a few seconds, as will be discussed later).

The fact that the channel is not time-varying implies that, in power/diversity gain MIMO configurations, only power gain effects can be observed. However, this is not critical, because diversity gain is simply a result of power gain in a fading channel.

The experiments proposed in this section use the Alamouti scheme for transmission and MRC for reception. The transmitted signal is comprised of passband pulses sent from $n_{\mathrm{t}}=2$ loudspeakers, and is received with $n_{\mathrm{r}}=2$ microphones. In order to facilitate time and phase synchronization, as well as channel estimation, the signal includes 2 pilot pulses at the beginning. The signal structure is as follows: 
1. First pilot pulse, transmitted from the left loudspeaker only;

2. Brief separation period, in which the transmitted signal is null;

3. Second pilot pulse, transmitted from the right loudspeaker only;

4. Brief separation period;

5. Sequence of modulated data pulses, transmitted from both loudspeakers with Alamouti pre-processing.

In the sequel, rows/columns " 1 " and " 2 " of $\mathbf{H}$ refer respectively to left and right microphones/loudspeakers. The carrier frequency will be denoted as $f_{\mathrm{c}}$, the pulse period as $T_{\mathrm{p}}$, the separation period as $T_{\mathrm{n}}$, and the total signal duration (including all pilot symbols, separation periods and data symbols) as $T$.

The purpose of the measurements is to provide an intuitive experience. This affects the choice of receiver operations and of the transmitted signal. Regarding the first, it is important that the students observe the received waveforms and deduce properties from them. This is preferable to simply giving a final result such as the sequence of demodulated symbols. As for the transmitted signal, simultaneously transmitting in the I and Q branches makes it difficult to visually recognize features in the received signal. Thus it may be advisable to transmit using only the I branch with unipolar pulses. On the other hand, I/Q modulations can also be used to demonstrate that the presented techniques are applicable in the general case.

All signal processing operations, both at the transmitter and at the receiver, are based on discrete-time signals. These are converted to/from continuous-time signals as part of the playing/recording processes. The sample rate will be denoted as $f_{\mathrm{s}}$.

In the following, transmitter and receiver processing are described. Restrictions on parameter values are then identified. Based on that, the values to be used in the measurements are selected. Finally, measurement results are presented and discussed.

\subsection{Transmitter and receiver processing}

Transmitter The transmitter first applies Alamouti spatial pre-processing on the complex data symbol sequence, as described in Section 2.1. It then builds the left and right lowpass equivalent waveforms including the pilot pulses, the latter without pre-processing. It subsequently generates the passband signals to be transmitted.

Before sending the passband signals to the loudspeakers, interpolation is applied to slightly change the sample rate, in order to compensate for small differences between transmitter and receiver clock rates. This will be discussed in Section 4.2.

Receiver The receiver processing begins by reading the stereo audio file that contains the two received signals. To these it applies a bandpass filter that eliminates noise outside the signal frequency band. This will be referred to as input filter.

The next step is to acquire time and phase synchronization. To this end, a sliding non-coherent correlator is applied to locate the first pilot pulse. The left received signal is arbitrarily chosen for this task. The signal is correlated with time-shifted I and $\mathrm{Q}$ versions of the passband pulse shape, and both results are quadratically combined. 
The time shifts span a search window of given length $T_{\mathrm{w}}$ starting at the beginning of the audio file. The position of the maximum correlation gives the desired time and phase reference.

Channel estimation is then carried out. Once a time and phase reference has been established for the pilot pulses, the I and Q correlators provide an estimate of $\mathbf{H}$, which will be denoted as $\hat{\mathbf{H}}$.

The received signal is then converted to a complex lowpass equivalent. This is possible because Matlab works with complex numbers; and it has the advantage that it simplifies subsequent processing. The standard technique is used: the passband signal is multiplied by $e^{j 2 \pi f_{\mathrm{c}}}$ and a lowpass filter is applied to eliminate the doublefrequency component. This filter will be referred to as frequency-conversion filter.

This is followed by Alamouti spatial post-processing. In a real system this would be applied to the unquantized symbols after demodulation. However, since in the present case the waveforms are to be directly displayed, the Alamouti post-processing has to be applied, interval-wise, to the left and right received signal waveforms.

As a result of Alamouti post-processing, assuming ideal estimation $\hat{\mathbf{H}}=\mathbf{H}$, the two received signals have equivalent-channel coefficients $\left|h_{1,1}\right|^{2}+\left|h_{1,2}\right|^{2}$ and $\left|h_{2,1}\right|^{2}+$ $\left|h_{2,2}\right|^{2}$ respectively, as seen in Section 2.1; and the fact that these coefficients are real implies that both signals are phase-aligned. In practice this is an approximation. The signals are then normalized and separated into I (real) and Q (imaginary) components.

The last step is MRC processing, which makes use of the known (estimated) equivalent-channel coefficients to obtain the combined signal.

\subsection{Restrictions on parameter values}

Sample rate The sample rate should satisfy $f_{\mathrm{s}} \gg f_{\mathrm{c}}+1 / T_{\mathrm{p}}$ so that the Nyquist criterion is amply fulfilled. This assures that a simple plot of the sampled values provides a good representation of the underlying waveform. Modern sound cards use sample rates of the order of $10^{4}-10^{5}$ samples/s, which is sufficient for all purposes.

Input filter Since the received signal is to be observed rather than demodulated, the input filter should be sufficiently flat across the signal bandwidth to avoid distortion; and should have a bandwidth $W_{\mathrm{i}}$ slightly larger than that of the signal. This condition can be expressed as $W_{\mathrm{i}} \approx 2 / T_{\mathrm{p}}$, where the factor 2 is admittedly somewhat arbitrary.

Frequency-conversion filter The lowpass filter used in the frequency conversion should also be flat across the signal bandwidth, and should have a cutoff frequency $W_{\mathrm{f}}$ such that $2 / T_{\mathrm{p}}<W_{\mathrm{f}} \ll 2 f_{\mathrm{c}}$. Standard Butterworth and Chebyshev designs tend to alter the shape of the observed time-domain pulses asymmetrically, that is, they affect the rising and falling flanks differently. To avoid this, a solution is to use the filter twice in a time-symmetric fashion: it is applied first to the input signal, and then to the time-reversed version of the result (using Matlab's filtfilt function).

Mains electricity coupling Mains electricity produces spectral components at the fundamental frequency $f_{\mathrm{m}}(50$ or $60 \mathrm{~Hz})$ and its harmonics. These should be eliminated by the input filter. The condition for this is $\left|f_{\mathrm{c}}-k f_{\mathrm{m}}\right| \gg 1 / T_{\mathrm{p}}$ for all $k \in \mathbb{N}$. 
Difference in clock rates Clock rates of sound cards typically differ by several tens of ppm [3]. To compensate for this, either the transmitted or the received signal needs to be resampled. The required resampling factor can be determined by transmitting a long test signal and comparing with the received signal.

The method used in [3] was based on Matlab's resample function. Due to limitations with this function, the precision in the correction is about $10 \mathrm{ppm}$. The residual rate difference gives rise to a phase error that accumulates over time. This was not important in the cited reference, where only phase differences between multipath components at a given time mattered. However, in the present case, the accumulated phase error may cause part of the I signal to couple to the $\mathrm{Q}$ branch, and vice versa.

Consider a received signal of duration $T$ and carrier frequency $f_{\mathrm{c}}$. Assume that after clock rate compensation there is a small residual error, such that the compensated clock rate at the receiver is $(1+\varepsilon)$ times the clock rate at the transmitter. Then the accumulated phase shift at the end of the signal will be $T f_{\mathrm{c}} \varepsilon$, measured in turns. It has been observed that a phase error of the order of $1 / 100$ turn is just enough to produce visible coupling. Thus the condition for $\varepsilon$ is $T f_{\mathrm{c}} \varepsilon<0.01$. For $T=10 \mathrm{~s}$ and $f_{\mathrm{c}}=300 \mathrm{~Hz}$ (these values are similar to those that will be used in the measurements; see Section 4.3 below), this means that $\varepsilon$ should be smaller than $\pm 3.3 \mathrm{ppm}$.

To achieve this, the transmitted signal will be resampled using linear interpolation (Matlab's interp1 function). This allows adjusting the resampling factor very finely, and the introduced distortion is negligible for resampling factors close to 1 .

Delay spread of the propagation channel If the delay spread of the channel, $\tau_{\mathrm{s}}$, is comparable to or larger than the pulse period, inter-symbol interference will be noticeable and will make it difficult to observe the MIMO-related features in the received signal. To avoid this it is necessary that $\tau_{\mathrm{s}} \ll T_{\mathrm{p}}$. Using a smooth pulse shape also helps make the effects of this interference less evident.

Delay spreads of a few tenths of a second should be expected in a typical classroom. Thus $T_{\mathrm{p}}$ values of at least 1 second are advised. This restricts the number of transmitted symbols, so as to avoid an excessive signal duration $T$.

Separation period The synchronization procedure uses a search window of width $T_{\mathrm{w}}$ (see Section 4.1). A large value of $T_{\mathrm{w}}$ would be desirable. However, $T_{\mathrm{p}}+T_{\mathrm{n}}$ needs to be greater than $T_{\mathrm{w}}$, otherwise the second pilot pulse could be mistaken for the first. Thus using a large $T_{\mathrm{w}}$ increases the total duration $T$. Therefore, once $T_{\mathrm{p}}$ has been selected, $T_{\mathrm{w}}$ should be chosen as a compromise value, and $T_{\mathrm{n}}>T_{\mathrm{w}}-T_{\mathrm{p}}$.

Acceleration of synchronization procedure To reduce computation time, the sliding correlation is carried out on a decimated version of the signal. As a result, the precision of the resulting time reference cannot be better than $D / f_{\mathrm{s}}$, where $D$ is the decimation factor. However, the actual accuracy of the correlation is determined by pulse duration and shape, which will be the limiting factors as long as $D / f_{\mathrm{s}} \ll T_{\mathrm{p}}$. 
Table 1: Restrictions on parameter values

\begin{tabular}{|l||l|}
\hline Sample rate & $f_{\mathrm{s}} \gg f_{\mathrm{c}}+1 / T_{\mathrm{p}}$ \\
\hline Input filter (bandpass) & $W_{\mathrm{i}} \approx 2 / T_{\mathrm{p}}$ \\
\hline Frequency-conversion filter (lowpass) & $2 / T_{\mathrm{p}}<W_{\mathrm{f}} \ll 2 f_{\mathrm{c}}$ \\
& Smooth pulse shape \\
\hline Mains electricity & $\left|f_{\mathrm{c}}-k f_{\mathrm{m}}\right| \gg 1 / T_{\mathrm{p}}$ for all $k \in \mathbb{N}$ \\
\hline Clock rate difference & $T f_{\mathrm{c}} \varepsilon<0.01$ \\
\hline Channel delay spread & $\begin{array}{l}\tau_{\mathrm{s}} \ll T_{\mathrm{p}} \\
\text { Smooth pulse shape }\end{array}$ \\
\hline Separation period & $T_{\mathrm{n}}>T_{\mathrm{w}}-T_{\mathrm{p}}$ \\
\hline Acceleration of synchronization & $D / f_{\mathrm{s}} \ll T_{\mathrm{p}}$ \\
\hline
\end{tabular}

Table 2: Selected parameter values

\begin{tabular}{|l||l|}
\hline Sample rate, $f_{\mathrm{s}}$ & 48000 samples/s \\
\hline Carrier frequency, $f_{\mathrm{c}}$ & $320 \mathrm{~Hz}$ \\
\hline Pulse period, $T_{\mathrm{p}}$ & $1 \mathrm{~s}$ \\
\hline Pulse shape & Time-domain raised cosine \\
\hline Input filter (bandpass) & Butterworth; order $2 ;$ \\
& $3-\mathrm{dB}$ passband: $\left[f_{\mathrm{c}}-4 / T_{\mathrm{p}}, f_{\mathrm{c}}+4 / T_{\mathrm{p}}\right]$ \\
\hline Frequency-conversion filter (lowpass) & Butterworth; order $1 ;$ \\
& 3 -dB cutoff frequency: $4 / T_{\mathrm{p}}$ \\
\hline Separation period, $T_{\mathrm{n}}$ & $0.3 \mathrm{~s}$ \\
\hline Signal duration, $T$ & $8.6 \mathrm{~s}(6$ data symbols) \\
\hline Downsampling factor, $D$ & 10 \\
\hline
\end{tabular}

\subsection{Selected parameter values}

Parameter restrictions are summarized in Table 1. From this, the values given in Table 2 are selected. A time-domain raised cosine pulse shape is used, given as $1-\cos \left(2 \pi t / T_{\mathrm{p}}\right), 0 \leq t \leq T_{\mathrm{p}}$. Small-order Butterworth designs are used for the filters.

\subsection{Example measurements}

The measurements to be presented have been made in an office room using the values in Section 4.3 (see Table 2). Two cases are considered: in case I a signal with inphase, unipolar pulses is used. In case II two bipolar, I/Q-multiplexed sequences are employed. The first case lends itself to simple, visual interpretation; whereas the second is closer to the modulation schemes used in real systems. Table 3 defines all measurement cases (including those that will be described in the following section).

In case I (in-phase, unipolar), the following sequence $x[m]$ of 6 data symbols is used: $1,0,1,0,1,0$. Using the notation of Section 2.1, the sequences $\bar{x}_{1}[\mathrm{~m}]$ and $\bar{x}_{2}[m]$ after Alamouti pre-processing are respectively 1,0,1,0,1,0 and $0,1,0,1,0,1$. Figure 4 shows the left and right received signals as recorded by the audio editing program. In all figures, the horizontal axis represents time in seconds. Comparing 
Table 3: Measurement cases

\begin{tabular}{|l||l|l|l|}
\hline Case & MIMO technique & I/Q multipl. & Pulse polarity \\
\hline I & Power gain: Alamouti and MRC & No & Unipolar \\
\hline II & Power gain: Alamouti and MRC & Yes & Bipolar \\
\hline III & Spatial multipl.: V-BLAST & Yes & Bipolar \\
\hline IV & Spatial multipl.: SVD & Yes & Bipolar \\
\hline
\end{tabular}

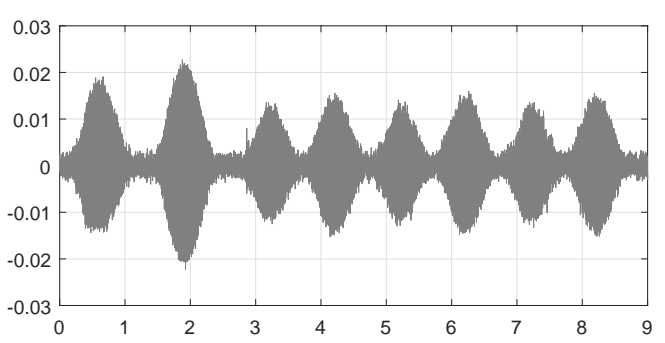

(a) Left receiver

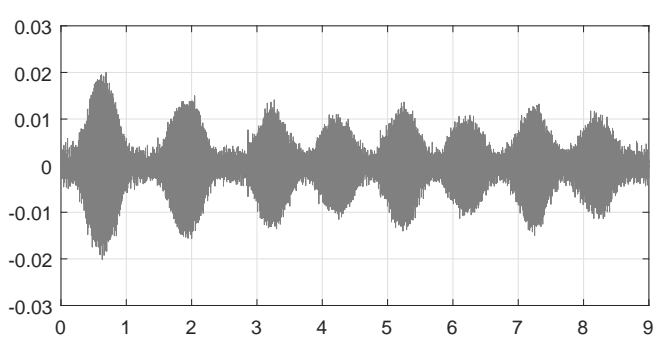

(b) Right receiver

Figure 4: Received signals, case I (Alamouti/MRC)

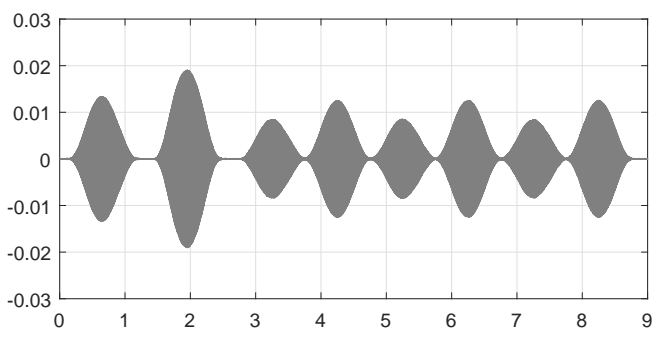

(a) Left receiver

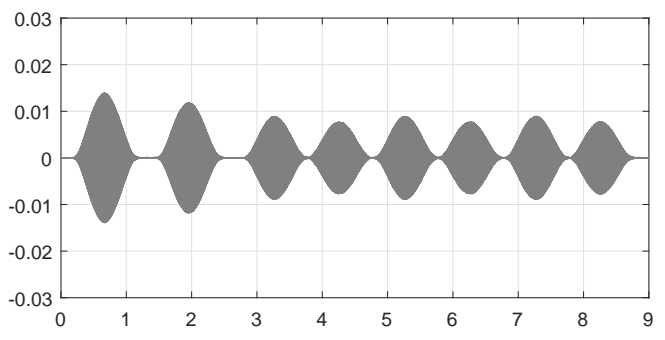

(b) Right receiver

Figure 5: Received signals after input filter, case I (Alamouti/MRC)

Figure 4 with $\bar{x}_{1}[m]$ and $\bar{x}_{2}[m]$, it is clear that each microphone receives a mixture of the left and right transmitted signals, and thus $\bar{x}_{1}[m]$ and $\bar{x}_{2}[m]$ cannot be directly recognized in the figure.

The signals after the input filter are shown in Figure 5. Note how most of the noise has been eliminated. This is because the signal bandwidth (a few $\mathrm{Hz}$ ) is much smaller than the total audio bandwidth (about $20 \mathrm{kHz}$ ). The first two pulses are the pilot pulses (note also the 0.3-s separation periods). From these, the estimated channel matrix $\hat{\mathbf{H}}$, normalized to $0 \mathrm{~dB}$ maximum amplitude and taking $\hat{h}_{1,1}$ as the phase reference, is

$$
\hat{\mathbf{H}}=\left(\begin{array}{cc}
-3.2 \mathrm{~dB} \angle 0^{\mathrm{o}} & 0 \mathrm{~dB} \angle-53^{\circ} \\
-2.9 \mathrm{~dB} \angle-142^{\circ} & -4.2 \mathrm{~dB} \angle-101^{\circ}
\end{array}\right) .
$$

It can be checked that the moduli of the matrix elements match the relative pilot amplitudes in Figure 5 (first row of $\hat{\mathbf{H}}$ corresponds to left receiver, second to right).

The baseband signals after Alamouti post-processing are shown in Figure 6. These correspond to sequences $y_{1}[m]$ (left) and $y_{2}[m]$ (right) in Section 2.1, normalized so that the peak squared amplitudes of the four components (left/right, I/Q) sum to 2. Solid blue and dashed red lines correspond to the I and Q branches respectively. The 


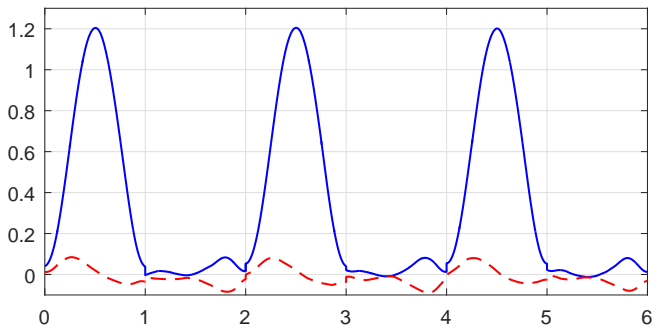

(a) Left receiver

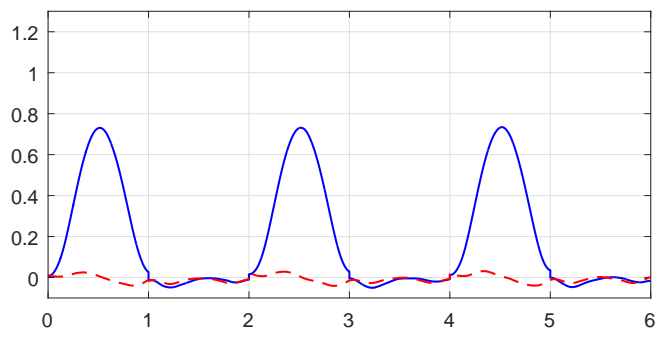

(b) Right receiver

Figure 6: Baseband signals after Alamouti post-processing, case I (Alamouti/MRC)

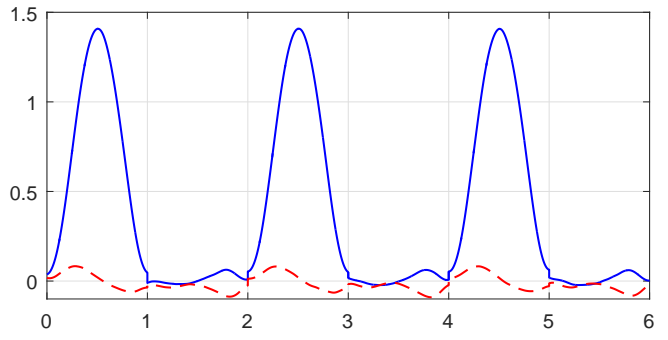

Figure 7: Baseband signal after MRC, case I (Alamouti/MRC)

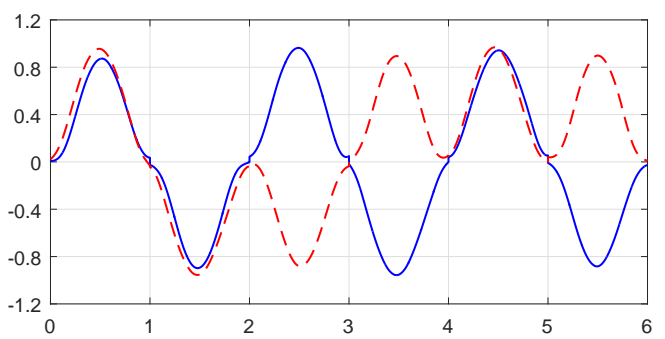

(a) Left receiver

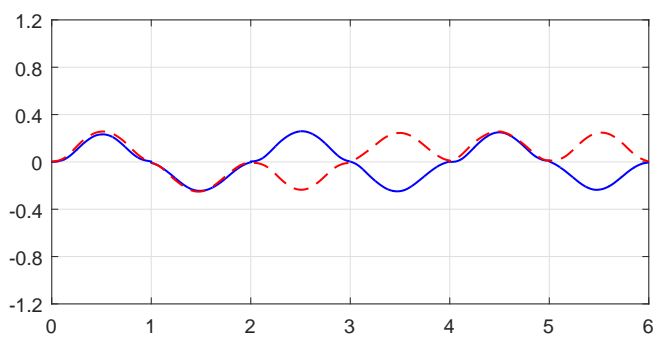

(b) Right receiver

Figure 8: Baseband signals after Alamouti post-processing, case II (Alamouti/MRC)

unipolar pulses representing the data sequence are clearly visible in the I branch of the left and right receivers. Some noise and slight I/Q coupling are also seen.

Lastly, Figure 7 displays the I and Q signals after MRC, corresponding to sequence $y[m]$ in Section 2.1. Again, the original data sequence is easily recognized.

Case II (I/Q, bipolar) uses the same in-phase sequence as before and the quadrature sequence $1,0,0,1,1,1$. Since bipolar modulation is used on each branch, with 0 transformed into -1 , this produces $\operatorname{Re} x[m]$ and $\operatorname{Im} x[m]$ given as $+1,-1,+1,-1,+1,-1$ and $+1,-1,-1,+1,+1,+1$ respectively. Figures 8 and 9 are analogous to Figures 6 and 7 (in case II the filtered received signals and estimated channel matrix are omitted for brevity). The two original sequences are clearly identified.

\section{Spatial multiplexing measurements}

The measurements in this section illustrate spatial multiplexing of $n=2$ streams, with $n_{\mathrm{t}}=n_{\mathrm{r}}=2$. Two functioning modes are considered: V-BLAST and SVD, as 


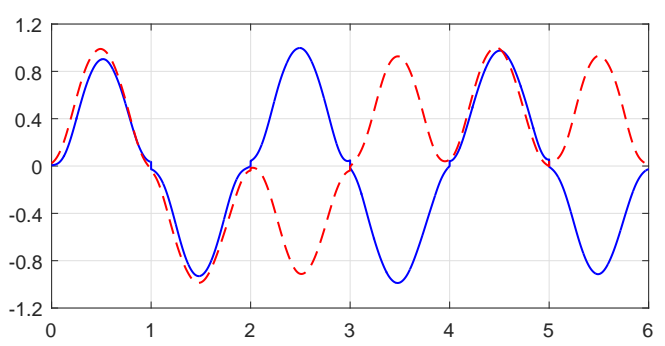

Figure 9: Baseband signal after MRC, case II (Alamouti/MRC)

described in Section 2.2. The signal structure is the same as in Section 4, only the data part consists of 2 spatial streams.

The propagation channel is required to be static for the duration of each measurement, as indicated in Section 4. Additionally, in the SVD mode the channel needs to remain unchanged between two consecutive measurements, because this mode uses channel information fed back from a previous measurement, as will be detailed later.

\subsection{Transmitter and receiver processing}

The transmitter and receiver operations are similar to those in Section 4.1. Only the spatial pre-processing and post-processing are different.

V-BLAST mode The transmitter applies $\mathbf{P}_{\mathrm{t}}=\mathbf{I}$ (see Section 2.2). The receiver obtains the channel matrix estimate $\hat{\mathbf{H}}$ and applies $\mathbf{P}_{\mathrm{r}}=\hat{\mathbf{H}}^{-1}$, assuming $\hat{\mathbf{H}}$ non-singular.

Although not properly part of this mode, the receiver also computes and stores the SVD decomposition of $\hat{\mathbf{H}}$ (matrices $\hat{\mathbf{U}}, \hat{\mathbf{V}}$ and $\hat{\Lambda}$ ), to be used in the SVD mode.

SVD mode The transmitter uses $\mathbf{P}_{\mathrm{t}}=\hat{\mathbf{V}}$, where $\hat{\mathbf{V}}$ has been computed by the receiver in a previous run and fed back to the transmitter (in the form of a Matlab data file). The receiver applies a first stage of spatial post-processing using $\mathbf{P}_{\mathrm{r}}=\hat{\mathbf{U}}^{*}$. The pilot symbols also undergo the pre-processing and the first post-processing. This is done in order to facilitate the second stage of post-processing, described next.

The pre-processing and first post-processing transform the channel $\mathbf{H}$ into a composite channel $\mathbf{H}^{\prime}=\hat{\mathbf{U}}^{*} \mathbf{H} \hat{\mathbf{V}}$. Ideally this would achieve total decoupling of the spatial streams. In practice the channel estimation is subject to errors, and as a result $\mathbf{H}^{\prime}$ is not exactly diagonal. Thus, a second stage of post-processing is applied, which treats $\mathbf{H}^{\prime}$ as a channel to be diagonalized. An estimation $\hat{\mathbf{H}}^{\prime}$ is obtained and its inverse is used to decorrelate the streams. Estimation of the composite channel is straightforward thanks to the fact that the matrices $\hat{\mathbf{V}}$ and $\hat{\mathbf{U}}^{*}$ have been applied to the pilot symbols.

\subsection{Restrictions and selected parameter values}

The parameter restrictions are the same as in the power gain measurements (Section 4.2). Accordingly, the same parameter values as in Table 2 are used. 


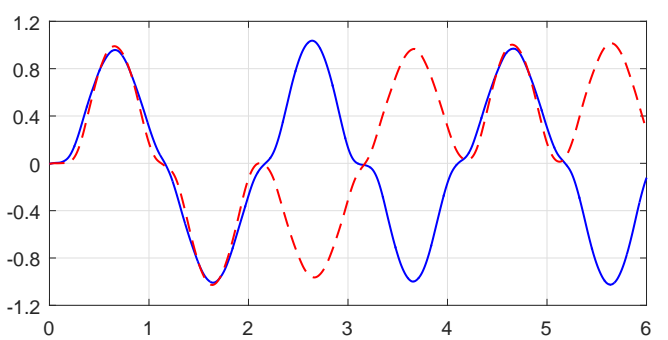

(a) Stream 1

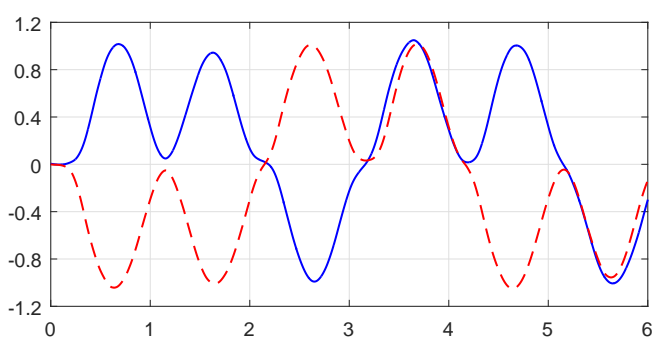

(b) Stream 2

Figure 10: Baseband signals after spatial post-processing, case III (V-BLAST)

\subsection{Example measurements}

The propagation environment is similar to that of Section 4.4. Two measurement cases will be considered: one with V-BLAST and one with SVD, both using I/Q, bipolar pulses. These are defined in Table 3 as cases III and IV respectively.

Case III (V-BLAST) uses for the first spatial stream the same data sequences as case II, that is, 1,0,1,0,1,0 in the I branch and 1,0,0,1,1,1 in the Q branch. Using the notation of Section 2.2 with time index $m$, the real and imaginary parts of $x_{1}[m]$ are respectively given by the bipolar forms of these sequences. The second stream $x_{2}[m]$ has data sequences $1,1,0,1,1,0$ and $0,0,1,1,0,0$ in the I and $\mathrm{Q}$ branches, again transformed into bipolar values. The normalized channel matrix estimated from the pilot symbols is as follows (the filtered received signals are omitted for brevity):

$$
\hat{\mathbf{H}}=\left(\begin{array}{cr}
-1.3 \mathrm{~dB} \angle 0^{\mathrm{o}} & 0 \mathrm{~dB} \angle-42^{\circ} \\
-2.3 \mathrm{~dB} \angle-129^{\circ} & -4.1 \mathrm{~dB} \angle-94^{\circ}
\end{array}\right) .
$$

The post-processed baseband signals, corresponding to the two spatial streams, are shown in Figure 10. The I and Q parts of the original sequences $x_{1}[\mathrm{~m}]$ and $x_{2}[\mathrm{~m}]$ can be clearly identified. No coupling is observed between the signals.

The measurements for case IV (SVD) are made in exactly the same physical scenario as in case III, so that the channel matrix is the same. The SVD of the estimated matrix $\hat{\mathbf{H}}$ given above (case III) is first computed. The condition number turns out to be 2.91, which indicates that, although stream 1 is (by definition) stronger than stream 2, the difference in SNR will not be very large. The matrix $\hat{\mathbf{V}}$ is manually fed back to the transmitter for the pre-processing to be applied, whereas the receiver uses $\hat{\mathbf{U}}^{*}$ for the first post-processing. After the first post-processing at the receiver, the normalized estimated matrix of the composite channel is

$$
\hat{\mathbf{H}}^{\prime}=\left(\begin{array}{cc}
0 \mathrm{~dB} \angle 0^{\circ} & -32.0 \mathrm{~dB} \angle-10^{\circ} \\
-37.4 \mathrm{~dB} \angle 140^{\circ} & -8.7 \mathrm{~dB} \angle 7^{\circ}
\end{array}\right),
$$

which is seen to be nearly diagonal. After the second post-processing, the baseband signals shown in Figure 11 are obtained. Again, the original complex sequences $x_{1}[\mathrm{~m}]$ and $x_{2}[m]$ are easily recognized. Both spatial streams are received without appreciable noise (clean graphs, data sequences clearly identifiable from the signals). Had the condition number been large, the weakest stream would have been more degraded. 


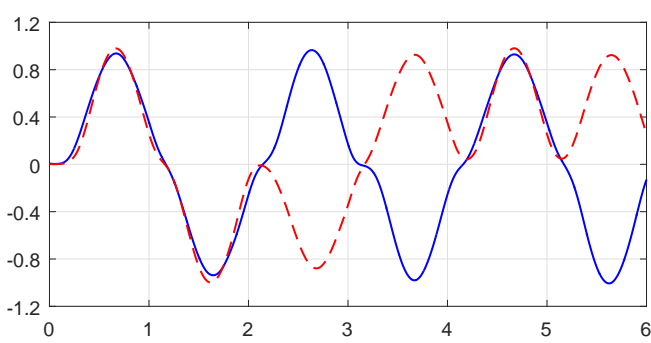

(a) Stream 1

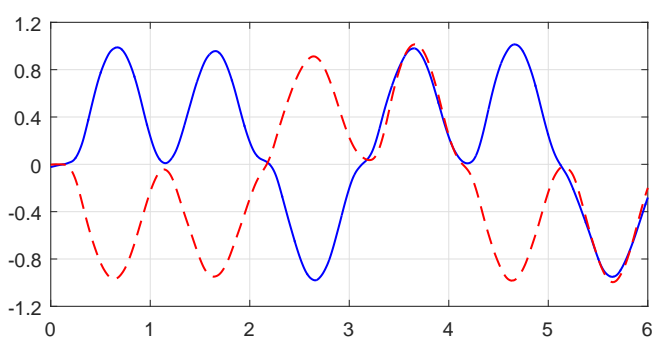

(b) Stream 2

Figure 11: Baseband signals after second spatial post-processing, case IV (SVD)

To see the impact of the condition number, the measurements of cases III and IV are repeated in a different scenario, with the two microphones closer together. ${ }^{5}$ By testing several positions, it is observed that the condition number increases as the microphones approach each other. This was expected, because a reduced distance between the receivers implies greater similarity between the rows of $\mathbf{H}$, which then becomes closer to singular.

The results below are obtained for a specific placement of the microphones that results in a large condition number. In that scenario, the measurements for cases III and IV are repeated with decreasing levels of signal amplitude: $0 \mathrm{~dB},-10 \mathrm{~dB}$ and -20 $\mathrm{dB}$. Here $0 \mathrm{~dB}$ represents a reference amplitude which is similar to those in previous measurements. No attempt has been made to estimate the actual received SNR, and thus these measurements can only be interpreted in a relative sense, i.e. they indicate to what extent the received signal quality is degraded when the SNR is decreased.

Figures 12 and 13 show the results. The obtained condition number is 27.3. With V-BLAST both spatial streams degrade similarly, whereas in the SVD case there is a very noticeable difference in quality between streams 1 (strongest) and 2 (weakest).

\section{Conclusions and possible extensions}

This paper has shown how the fundamental concepts of MIMO transmission can be illustrated using cheap equipment. Features that can be observed include:

- the essential role of channel estimation, both for power gain/diversity and for spatial multiplexing; and the ability of all presented techniques to recover the original symbols using non-ideal channel estimates;

- the difference between V-BLAST and SVD-based spatial multiplexing, in terms of functioning and necessity of channel information feedback;

- the impact of condition number on each spatial multiplexing mode.

The measurements can be easily extended to cover other topics, such as how performance is degraded when channel estimation errors are larger. This can be done by gradually reducing the amplitude of the pilot pulses. Another aspect that can be observed is the impact of time variation: by using a long sequence of data symbols

\footnotetext{
${ }^{5}$ Placing the loudspeakers closer to each other would have a similar effect.
} 


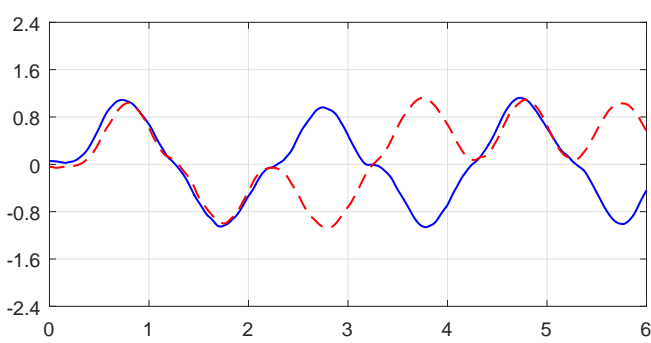

(a) 0 - $\mathrm{dB}$ reduction, stream 1

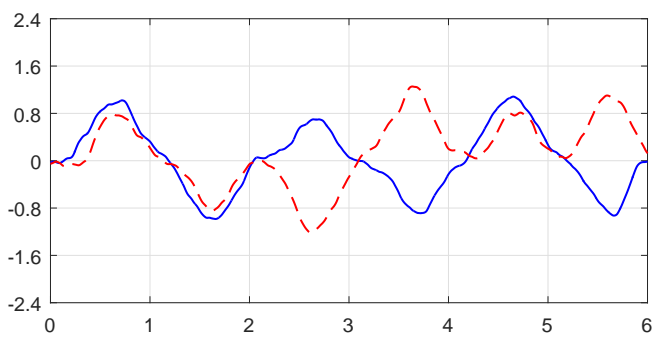

(c) $10-\mathrm{dB}$ reduction, stream 1

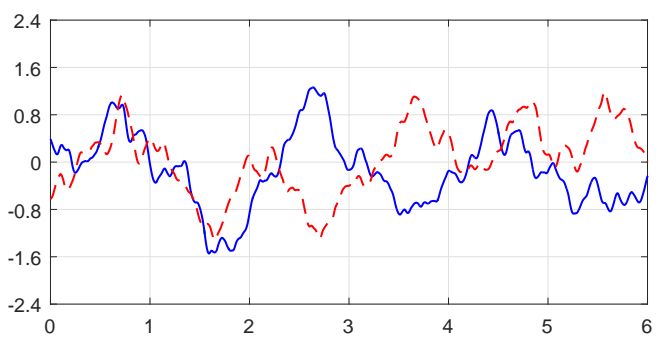

(e) 20-dB reduction, stream 1

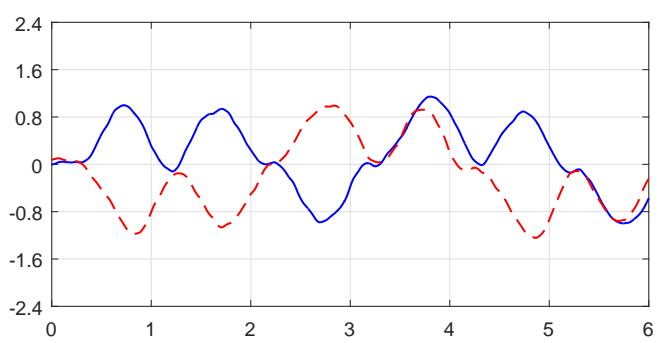

(b) 0-dB reduction, stream 2

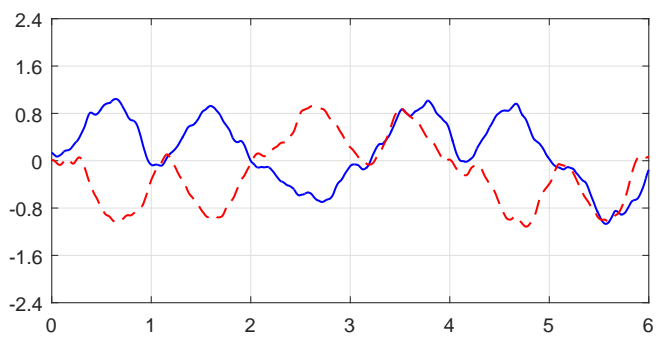

(d) $10-\mathrm{dB}$ reduction, stream 2

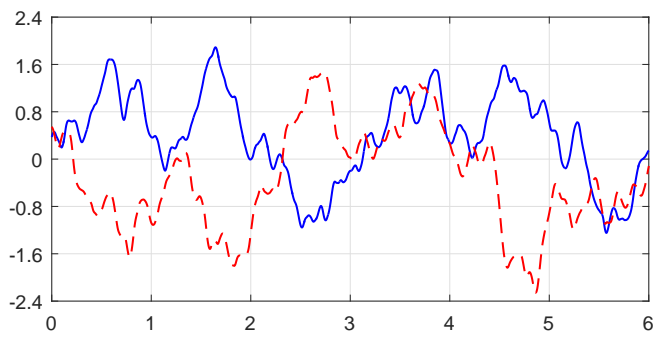

(f) $20-\mathrm{dB}$ reduction, stream 2

Figure 12: Baseband signals after spatial post-processing, case III (V-BLAST) with reduced SNR

and slowly moving the microphones during the measurement, signal reception will be more degraded for symbols located farther away from the pilot pulses.

\section{Acknowledgment}

The author thanks Prof. P. García del Pino for providing some of the audio hardware, and the anonymous reviewers and Associate Editor for constructive comments.

\section{References}

[1] Siavash M. Alamouti. A simple transmit diversity technique for wireless communications. IEEE Journal on Selected Areas in Communications, 16(8):14511458, October 1998.

[2] Erik Dahlman, Stefan Parkvall, Johan Sköld, and Per Beming. $3 G$ Evolution. HSPA and LTE for Mobile Broadband. Academic Press, second edition, 2008. 


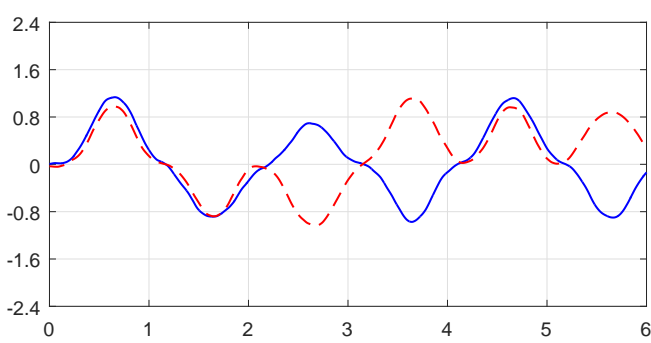

(a) $0-\mathrm{dB}$ reduction, stream 1

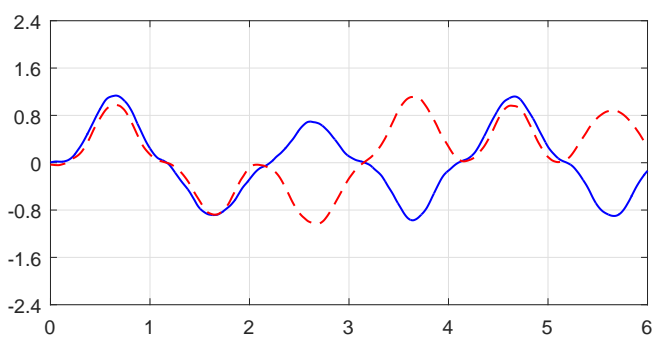

(c) 10-dB reduction, stream 1

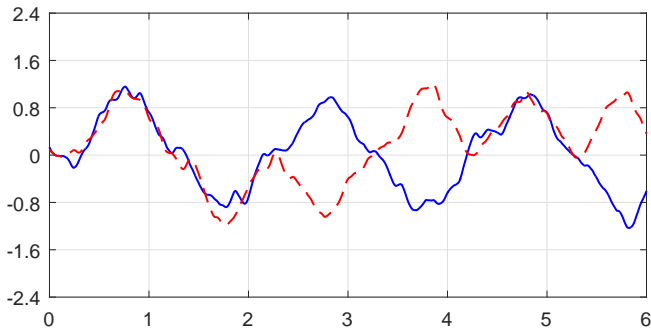

(e) 20-dB reduction, stream 1

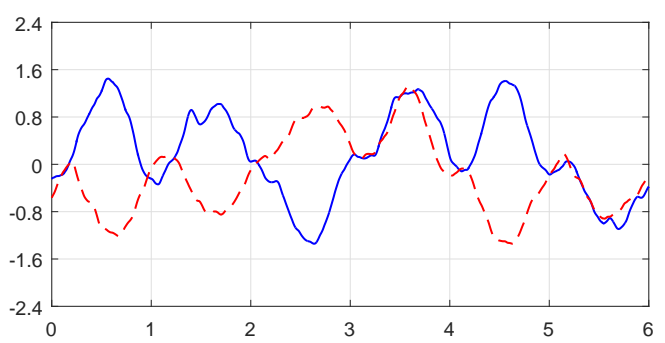

(b) 0-dB reduction, stream 2

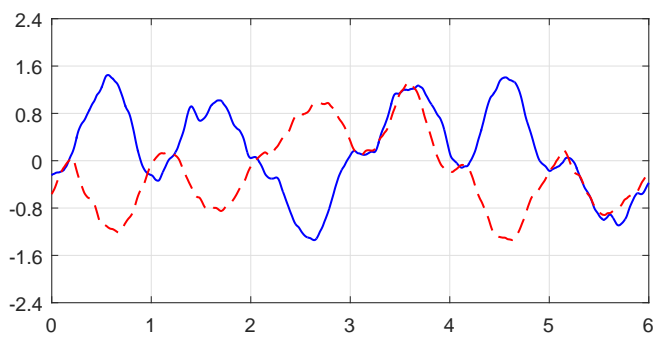

(d) 10-dB reduction, stream 2

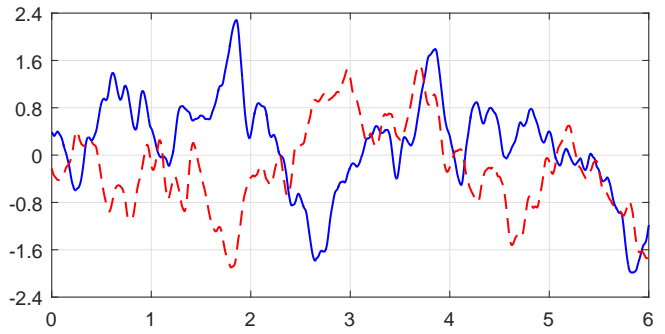

(f) 20-dB reduction, stream 2

Figure 13: Baseband signals after second spatial post-processing, case IV (SVD) with reduced SNR

[3] Luis Mendo. Illustrating multipath propagation by means of sound waves. IEEE Antennas and Propagation Magazine, 51(4):173-184, August 2009.

[4] David Tse and Pramod Viswanath. Fundamentals of Wireless Communication. Cambridge University Press, 2005. 